\title{
MEDICIONES DE VIBRACIONES AMBIENTALES EN TRES EDIFICIOS DE CONCRETO REFORZADO DE 28, 11 Y 6 PISOS
}

\author{
Luis Carlos Esquivel Salas ${ }^{(1)}$ y Víctor Schmidt Díaz ${ }^{(1)}$
}

\begin{abstract}
RESUMEN
Se presentan los resultados de cinco mediciones de vibraciones ambientales realizadas en tres distintos edificios de San José, Costa Rica. El objetivo principal fue el medir la mayor cantidad de periodos naturales en cada dirección de los edificios utilizando la técnica "peak-picking" con espectros de Fourier (EF) y funciones de transferencia (FT). También se calcularon los amortiguamientos modales para uno de los edificios por medio de la técnica de decaimiento aleatorio. Para los edificios estudiados, se concluye que las diferencias entre los periodos naturales calculados a partir de EF y FT son despreciables, así mismo para cada edificio, basta contar con dos puntos de medición, uno en el nivel inferior y otro en el superior para lograr identificar al menos cuatro periodos naturales. Para el caso del edificio de 28 pisos, se concluye que su nivel de operación no afectó la identificación de los periodos naturales. En el caso del edificio de 11 pisos, el avance constructivo provocó un cambio significativo en el periodo fundamental y amortiguamiento, únicamente para la dirección Norte-Sur. Para el edificio de 6 pisos se calibró un modelo matemático tomando en cuenta cargas gravitacionales reales, secciones no agrietadas de elementos estructurales, una rigidez infinita y restricción a la rotación en las conexiones viga-columna de todos los marcos.
\end{abstract}

Palabras clave: vibraciones ambientales; edificios; periodo natural; frecuencia; dinámica

\section{AMBIENT VIBRATION TESTS ON THREE REINFORCED CONCRETE BUILDINGS OF 28, 11 AND 6 FLOORS}

\begin{abstract}
The results from five ambient vibration tests performed on three different buildings in San José, Costa Rica, are presented. The main goal was to estimate as much natural periods as possible in both directions of the buildings using the peak picking technique with Fourier spectrums (EF) and transfer functions (FT). The damping was also estimated for one of the buildings using the random decrement technique. For the studied buildings, it is concluded that the differences of the natural periods estimated from EF and FT are negligible, also for each building, with just two measuring points, one in the bottom and the other on the top level, it is possible to identify at least four natural periods. For the 28 story building, it is concluded that its operational level didn't affect the natural period's identification. In the 11 story building, the construction progress caused a significant change in the fundamental period and its damping for the North-South direction. Finally, for the 6 story building a calibration of the mathematical model was perform taking into account the real gravitational loads, uncracked element sections, an infinite rigidity and a restriction to rotate on all frame beam-column connections.
\end{abstract}

Keywords: ambient vibrations; buildings; natural period; frequency; dynamics

Artículo recibido el 5 de febrero de 2016 y aprobado para su publicación el 21 de noviembre de 2016. Se aceptarán comentarios y/o discusiones hasta cinco meses después de su publicación.

(1) Laboratorio de Ingeniería Sísmica, Instituto de Investigaciones en Ingeniería, Universidad de Costa Rica, Sede Rodrigo Facio, San Pedro, San José, Costa Rica. luiscarlos.esquivel@ucr.ac.cr, victor.schmidt@ucr.ac.cr 


\section{INTRODUCCIÓN}

Al igual que cualquier estructura, los edificios vibran de acuerdo a sus propiedades dinámicas. Es importante poder caracterizar estos movimientos vibratorios con miras a modelar de manera acertada el comportamiento de la estructura bajo cargas que cambian instantáneamente con el tiempo, como por ejemplo las producidas por sismos o viento. Los patrones de vibración de un edificio son complejos, por lo que se descomponen en modos de vibración más sencillos, que superpuestos conforman la forma final en que vibrará la estructura. Cada uno de estos modos está definido por medio de tres parámetros: periodo natural $(T)$, amortiguamiento $(\beta)$ y forma modal.

Desde décadas atrás, estos parámetros dinámicos son medidos de manera experimental para piezas mecánicas de tamaños relativamente pequeños, a esto se le conoce como Análisis Modal Experimental (AME). La limitante en tamaño se debe a que la pieza debía poder colocarse en el banco de pruebas de un laboratorio, donde las cargas aplicadas a estas fueran aisladas, controladas y medidas (Andersen, s.f.).

Para el caso de estructuras como los edificios, es obvio que su tamaño no permitiría realizar este tipo de pruebas para medir sus propiedades dinámicas. Es por esto que en años recientes se idearon otro tipo de pruebas, donde se pudieran determinar los parámetros dinámicos sin necesidad de aislar o medir las cargas aplicadas, sino solamente la respuesta. A esto se le llama Análisis Modal Operacional (AMO), otros nombres que se utilizan para denominar este método son: mediciones de vibraciones ambientales (MVA), análisis modal de solo respuesta, análisis de respuesta ambiental o análisis de ruido (Andersen, s.f.).

Las pruebas de AMO se caracterizan por realizarse in situ, no interrumpen el uso normal de la edificación y son del tipo no destructivas. Las cargas aplicadas a la estructura para que esta vibre se originan en el ambiente e incluyen el viento, los microsismos y las actividades humanas, tanto de operación del edificio como externas a él. La respuesta se mide por medio de sensores, comúnmente acelerógrafos, colocados en puntos estratégicos del edificio.

En este artículo se exponen los resultados de mediciones de vibraciones ambientales (MVA) realizadas a tres edificios de concreto reforzado. Para cada edificio se estudió un aspecto diferente, a saber, influencia del nivel de operación en la identificación modal, cambio en parámetros dinámicos en función del avance constructivo y la calibración de un modelo numérico a partir de resultados de estas pruebas.

Las MVA en distintas etapas constructivas son escasas, pudiendo mencionar el trabajo de Schuster et al. (1994), Skrinar y Strukelj (1996), Ventura y Schuster (1996) y Tamura et al. (2002). Por otro lado, Oliveira y Navarro (2010) estimaron los periodos fundamentales de edificios de concreto reforzado en Portugal por medio de MVA y se compararon con los derivados de modelos numéricos. Carvajal (2005), Domínguez (2012) y Málaga (2007) son ejemplos de estimación de parámetros dinámicos por medio de MVA.

Finalmente, se han realizado varios trabajos en la calibración de modelos numéricos de edificios con ayuda de los resultados de MVA, como el caso de Elyamani et al. (2017), donde se realizó una evaluación sísmica de la Catedral de Mallorca utilizando un modelo calibrado a partir de parámetros medidos por medio de mediciones de vibraciones ambientales. Almac et al. (2016) calibró un modelo numérico de la torre de Macedonia en Edirne, Turquía, Foti et al. (2012) hace lo mismo para una torre histórica en Bari, Italia. Churilov et al. (2016) calibró un modelo de elemento finito de edificios de mampostería no reforzada en regiones sísmicas. Demirtas et al. (2016) comparó los resultados de un modelo numérico de un edificio alto ( sin calibrar con resultados de MVA) contra uno calibrado por medio de MVA, similar al caso de Günaydin 
et al. (2016), quienes construyeron tres estructuras de $1.7 \mathrm{~m}$ de altura en concreto reforzado para modelarlas numéricamente y comparar resultados con modelos calibrados por MVA.

\section{DESCRIPCIÓN DE LAS MVA Y CARACTERIZACIÓN DE LOS EDIFICIOS}

Se utilizaron dos tipos de equipo sincronizados en el tiempo por medio de GPS. Uno que se trasladó a los edificios exclusivamente para realizar las pruebas, el cual se denominará equipo portátil y otro instalado permanentemente en uno de los edificios, al cual se referirá como equipo permanente.

El equipo portátil consistió de tres acelerógrafos marca Nanometrics, mientras que el equipo permanente consistió de cinco acelerógrafos marca Reftek. Las principales características de ambos se resumen en la tabla 1.

Tabla 1. Acelerógrafos utilizados en MVA.

\begin{tabular}{ccc}
\hline Marca & Nanometrics & Reftek \\
Modelo & Titan SMA & 130-SMA \\
Sensores & Triaxiales Tipo FBA & Triaxiales Tipo Estado Sólido \\
Frecuencia de muestreo & $200 \mathrm{~Hz}(0.005 \mathrm{~s})$ & $200 \mathrm{~Hz}(0.005 \mathrm{~s})$ \\
Escala completa (Full scale) & $4 \mathrm{~g}$ & $4 \mathrm{~g}$ \\
Componentes adicionales & GPS, batería y cable de red & GPS, batería, fuente de poder y cable de red \\
\hline
\end{tabular}

En el caso de las mediciones con el equipo portátil, los acelerógrafos se colocaron en un mismo eje vertical, en los puntos inferior, superior y más cercano a la mitad de la altura del edificio. Para cada prueba se utilizaron un total de tres ejes verticales, desplazando la totalidad de los acelerógrafos a cada eje. Lo anterior se ilustra en la figura 1, donde se muestra la elevación esquematizada de un edificio, un eje vertical imaginario trazado y la ubicación final de los acelerógrafos para la MVA. La ubicación en planta de los tres ejes verticales se mostrará más adelante, en la sección correspondiente a cada edificio.

Para el caso de mediciones con el equipo permanente se utilizó un solo eje vertical de medición, definido por la ubicación de los acelerógrafos en el edificio, los cuales habían sido colocados varios años antes de realizar esta investigación. El esquema es análogo al de la figura 1, con la única diferencia que en lugar de tres acelerógrafos a lo largo del eje se utilizaron cinco.

En la tabla 2 se indican las principales características de los edificios estudiados, nombrados como edificios E1, E2 y E3. En la tabla 3 se incluye la información correspondiente a instrumentación y las MVA. 


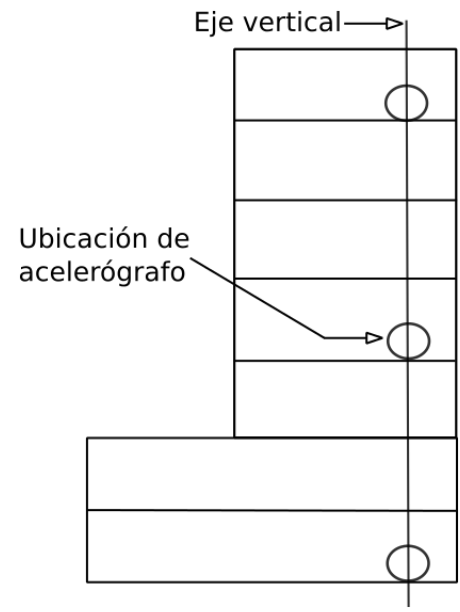

Figura 1. Esquema de ubicación de acelerógrafos

Tabla 2. Caracterización de los edificios estudiados.

\begin{tabular}{cccccccccc}
\hline Edificios & Sótanos & Pisos & Altura & Uso & SSR & Entrepiso & Cimentaciones & $\begin{array}{c}\text { Reg. } \\
\text { Planta }\end{array}$ & $\begin{array}{c}\text { Reg. } \\
\text { Altura }\end{array}$ \\
\hline E1 & 0 & 28 & 99 & Mixto Dual CR & PLCMP & PA+PiC & Sí & Sí \\
E2 & 2 & 11 & 48 & Mixto Dual CR & PLCMP & PA & No & No \\
E3 & 1 & 6 & 21 & Univ. Dual CR & PLCMP & PA + PiA & No & Sí \\
\hline
\end{tabular}

Uso mixto: comercio, oficinas y/o residencias.

Uso univ.: universidad, impartir clases, algunos laboratorios.

SSR dual CR: sistema sismo-resistente a base de muros y marcos de concreto reforzado.

Entrepiso PLCMP: entrepisos prefrabricados compuestos por losas de concreto multitubulares pretensadas con una sobrelosa colada en sitio.

Cimentaciones PA: placas aisladas unidas por vigas de fundación.

Cimentaciones PiC: pilotes de concreto.

Cimentaciones PiA: pilotes de acero.

Tabla 3. MVA e instrumentación por edificio.

\begin{tabular}{ccccccc}
\hline Edificios & $\begin{array}{c}\text { Cantidad de } \\
\text { MVA }\end{array}$ & $\begin{array}{c}\text { Ejes de } \\
\text { medición }\end{array}$ & $\begin{array}{c}\text { Ventana de } \\
\text { medición }\end{array}$ & Equipo & $\begin{array}{c}\text { Cantidad } \\
\text { Equipos }\end{array}$ & Ubicaciones \\
\hline E1 & 2 & 1 & 40 min & Permanente & 5 & $\begin{array}{c}\text { N0, N7, N15, } \\
\text { N21, N28 }\end{array}$ \\
E2 & 2 & 3 & 20 min & Portátil & 3 & S2, N5, N11 \\
E3 & 1 & 3 & 30 min & Portátil & 3 & SS, N3, N6 \\
\hline
\end{tabular}

N: nivel. S: sótano. SS: semi-sótano.

El E1 se encontraba terminado al $100 \%$ y en uso cuando se realizaron las mediciones de vibraciones ambientales (MVA), en dos condiciones de operación distintas con el objetivo de estudiar su efecto en la determinación de los parámetros dinámicos. Para esto, se seleccionaron dos horas diferentes del día, 3 am y 6 pm. Los registros obtenidos a las 3 am corresponden a un nivel de operación mínima del edificio, cuando 
se produce el menor tránsito interno de usuarios, tránsito externo de vehículos y la operación de máquinas o sistemas en la estructura se encuentran al mínimo. Por otra parte, los registros obtenidos a las $6 \mathrm{pm}$ corresponden al nivel de operación máximo del E1, donde las condiciones descritas para las 3 am son las opuestas.

En el E2 se realizaron dos MVA, la primera cuando el edificio se encontraba en obra gris, es decir, $0 \%$ de avance en acabados y elementos no estructurales, la segunda cuando el edificio contaba con un 50\% de avance en acabados y elementos no estructurales. Esto se hizo con el objetivo de cuantificar, con datos experimentales, el cambio en las propiedades dinámicas (periodo y amortiguamiento) producto del aporte de estos.

En el caso del E3 se realizó una única MVA de la cual se obtuvieron los parámetros dinámicos, el edificio se encontraba terminado al 100\%, sin embargo estaba desocupado. Se elaboró un modelo matemático de la estructura con ayuda del programa ETABS con el propósito de calibrarlo. Se utilizó como criterio el periodo fundamental, tratando de que el estimado por medio del modelo matemático fuera lo más parecido posible con el obtenido por medio de las MVA.

En la figura 2 se muestran las plantas típicas de los tres edificios estudiados, en las cuales se muestra la ubicación de cada eje de medición. Como se explicó anteriormente, estos ejes son verticales (perpendiculares al plano del dibujo) y la totalidad de los acelerógrafos en las MVA estaban alineados con los mismos.

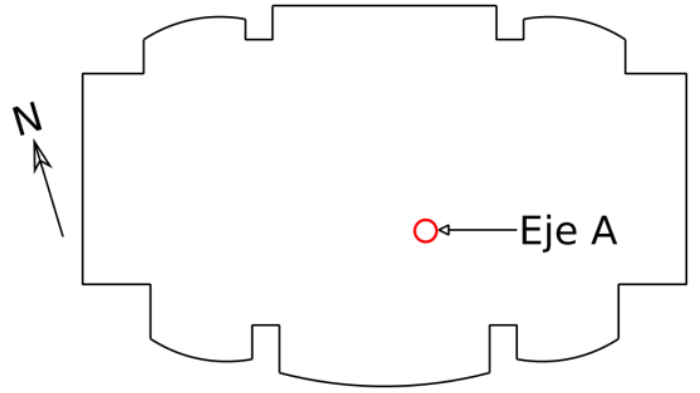

a) Edificio E1

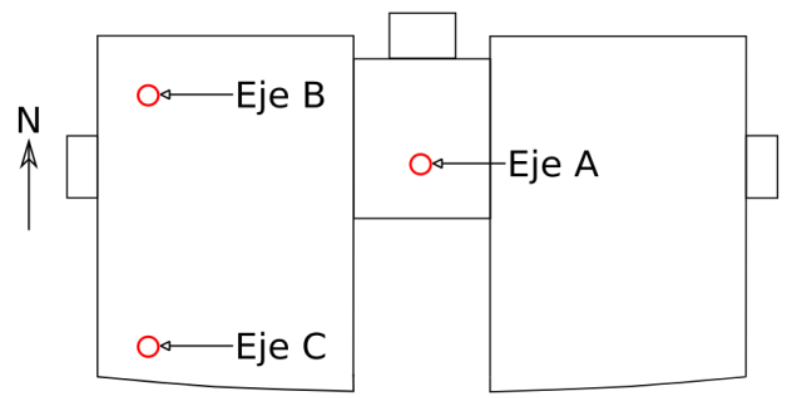

b) Edificio E2

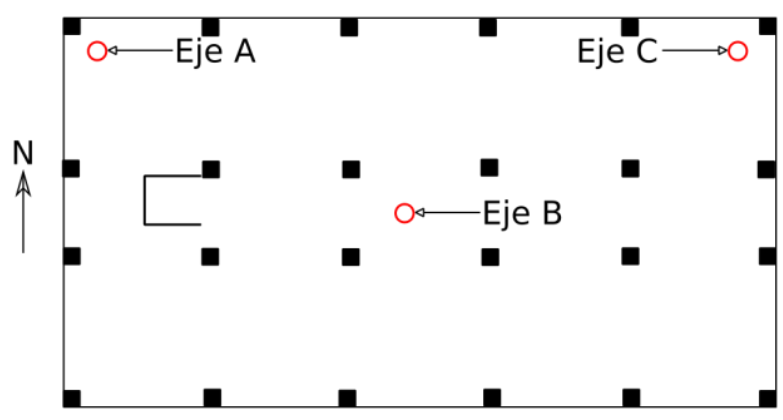

c) Edificio E3

Figura 2. Plantas típicas de edificios estudiados 


\section{METODOLOGÍA}

Para los tres edificios se siguió el mismo procedimiento para la estimación de sus periodos naturales en cada dirección. Los espectros de Fourier (EF) y las funciones de transferencia (FT) se calcularon utilizando la técnica "peak-picking" (Bendat y Piersol, 1993) con ayuda del software Geopsy (Wathelet, 2011). Básicamente, Geopsy aplica la transformada rápida de Fourier a una señal en el dominio del tiempo para convertirla al dominio de la frecuencia. Las amplitudes de cada frecuencia se relacionan a la cantidad de energía que aporta esa frecuencia a la señal, por lo que los picos en los espectros se asocian a las frecuencias naturales del sistema.

En el caso de los EF, estos se calcularon para cada punto de medición (cada ubicación de los acelerógrafos), con excepción de los ubicados en el nivel inferior, ya que los EF de estos no aportan información acerca de los periodos naturales de las estructuras. Sí lo hacen de otros aspectos de relevancia para otros análisis, como la interacción suelo-estructura o identificación de fuentes de excitación.

A manera de ejemplo se presenta un esquema en la figura 3, donde se muestra el caso para el E2, dirección Norte-Sur (NS). Cada uno de los puntos de medición se identifica por el eje al que pertenecen, la dirección de la medición y el nivel donde se ubica. Un punto de medición denominado A11 NS significa que el acelerógrafo estaba ubicado en el eje A (ver figura 2), nivel 11, midiendo en la dirección NS del edificio. Una vez obtenida la frecuencia natural para cada ubicación se procedió a calcular su inverso, que corresponde al periodo natural. Los periodos de cada eje se promediaron para obtener los periodos naturales por eje, los que a su vez se promediaron para obtener el periodo natural final promedio en esa dirección. La razón del paso intermedio (periodo natural por eje) es la de permitir la comparación entre periodos estimados por eje, con lo que se puede evaluar si hay diferencias significativas entre estos.

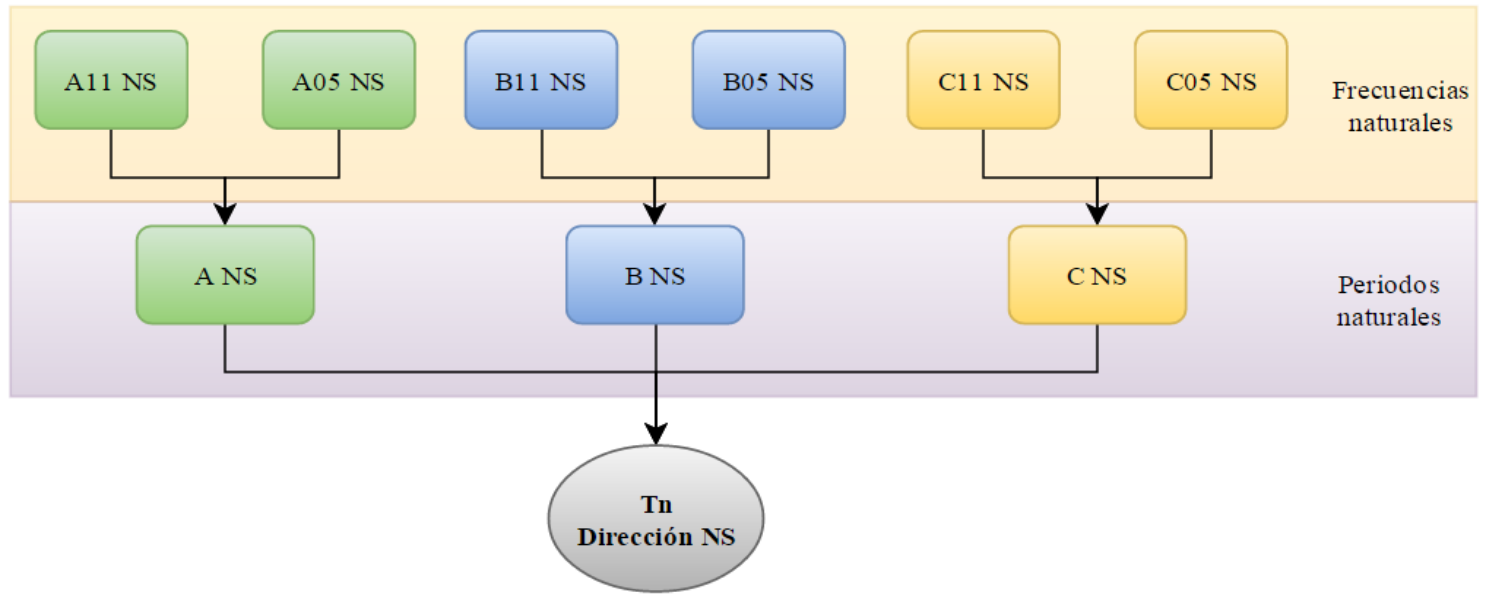

Figura 3. Periodos naturales promedio para EF

Para el caso de las FT se procedió de la misma manera, con la diferencia de que las FT calculadas son una razón entre el EF de un nivel intermedio dividido por el EF del nivel más bajo. Continuando con el ejemplo de la figura 3, el nombre de la FT entre los niveles 11 y de sótano, para el eje A dirección NS sería A11/Asot NS. Una vez obtenidos los periodos naturales para los EF y FT en cada edificio, se calcula la diferencia entre estos y se representa como un porcentaje del menor de los periodos. Con esto se pueden comparar muy rápidamente las estimaciones realizadas por medio de los EF y FT, con el fin de determinar si es posible obtener de manera confiable los periodos naturales de un edificio solamente a partir de EF. 
Para reflejar de una forma más representativa las diferencias entre los periodos naturales estimados por medio de los EF y las FT, estas se estimaron a manera de porcentaje correspondiente al menor de los periodos, de acuerdo a la ecuación 1.

$$
\frac{\operatorname{abs}(T . E F-T . F T)}{\min (T . E F, T . F T)} \times 100
$$

donde $T . E F$ es el periodo estimado con el EF, T.FT es el periodo estimado con la FT, $a b s$ es el valor absoluto de la operación en paréntesis, y mín es el mínimo de los valores en paréntesis.

Para evaluar las diferencias en las estimaciones de periodos naturales entre dos MVA en un mismo edificio (caso del E1 y E2) se utilizó la ecuación 2.

$$
\frac{T . E a-T . E b}{\min (T \cdot E a, T . E b)} \times 100
$$

donde $T . E a$ es el periodo correspondiente a la prueba Ea, y $T . E b$ es el periodo correspondiente a la prueba Eb. Un resultado negativo significa que el periodo estimado por la medición Eb fue mayor que el de Ea.

Para el cálculo del amortiguamiento modal se utilizó la técnica de decaimiento aleatorio (Asmussen, 1997; Rodrigues y Brincker, 2005), implementada por medio del software Geopsy. Básicamente, esta técnica asume que la señal medida contiene una parte aleatoria y otra correspondiente a la respuesta pura del sistema, en este caso un edificio. Se procede a seleccionar y promediar suficientes ventanas de análisis de la señal, con lo que disminuye hasta un nivel despreciable el aporte de la parte aleatoria en el promedio de las ventanas. Por último, a esta señal promedio se ajusta una función seno con decrecimiento exponencial de la cual se puede obtener la frecuencia y el amortiguamiento.

En el caso de esta investigación, la frecuencia estimada por esta técnica se utiliza únicamente para compararla con la estimada por los EF y FT y de esta manera decidir si el amortiguamiento calculado se valora como válido para el edificio en cuestión. En el caso de que la frecuencia determinada por la técnica de decaimiento aleatorio fuera significativamente diferente a la calculada por los EF y FT, el amortiguamiento correspondiente no se tomó como representativo.

Al igual que los EF, los amortiguamientos se calcularon en cada dirección para cada punto de medición, a excepción de los ubicados en los niveles inferiores. Luego se calculó un amortiguamiento por eje y por dirección, utilizando promedios como se explicó para el caso de los periodos naturales. Para el cálculo de los promedios se utilizaron los amortiguamientos señalados como válidos según el párrafo anterior.

\section{RESULTADOS Y ANÁLISIS}

En MVA las magnitudes de las aceleraciones medidas son muy bajas, como se comprobó en las cinco pruebas realizadas. Las aceleraciones ( $a$ prom) mínimas y máximas que aparecen en la tabla 4 son las obtenidas luego de aplicar un filtro pasa banda a los registros de $0.3 \mathrm{~Hz}$ a $30 \mathrm{~Hz}$. Se utilizaron estas frecuencias de esquina para el filtrado debido a que corresponden a los periodos de $3.3 \mathrm{~s}$ y $0.03 \mathrm{~s}$, asegurando de esta manera que los posibles periodos naturales de las estructuras no se vieran filtrados. Se muestran con unidades equivalentes a una fracción de la aceleración de la gravedad $\left(\mathrm{g}=9.81 \mathrm{~m} / \mathrm{s}^{2}\right)$. 
Tabla 4. Magnitud de aceleraciones en las MVA.

\begin{tabular}{ccccc}
\hline Edificio & Pisos & Altura máx $(\mathrm{m})$ & a prom mín $(\mathrm{g})$ & a prom máx $(\mathrm{g})$ \\
\hline E1 & 28 & 99 & $2 \times 10^{-5}$ & $2 \times 10^{-4}$ \\
E2 & 11 & 48 & $2 \times 10^{-5}$ & $3 \times 10^{-4}$ \\
E3 & 6 & 21 & $8 \times 10^{-6}$ & $2 \times 10^{-5}$ \\
\hline
\end{tabular}

En la tabla 4 se muestra lo pequeñas que son las magnitudes registradas en este tipo de prueba, del orden de $10^{-5} \mathrm{~g}$ y $10^{-4} \mathrm{~g}$. Las mismas son consistentes con las magnitudes reportadas en la literatura internacional; por ejemplo en el caso de Michel et al. (2008) se establece que estas aceleraciones oscilan entre $\operatorname{los} 10^{-7} \mathrm{~g}$ y $10^{-4} \mathrm{~g}$, mientras que Domínguez (2012) propone $\operatorname{los} 10^{-5} \mathrm{~g}$ y $10^{-4} \mathrm{~g}$. También se puede apreciar que el rango de las mismas es prácticamente igual para E1 y E2, mientras que E3 se diferencia ya que cuenta con magnitudes más pequeñas.

Para las siguientes secciones resulta práctico dar un nombre específico a cada prueba, como se detalla en la tabla 5. Adicionalmente, la nomenclatura que se utilizará para las direcciones respectivas de los edificios será la de NS para Norte-Sur y EO para Este-Oeste.

Tabla 5. Nomenclatura utilizada para las MVA realizadas.

\begin{tabular}{cccc}
\hline Edificios & Estado de edificio & MVA & Referencia \\
\hline \multirow{2}{*}{ E1 } & Terminado al 100\% y en uso. & 03:00 a. m. & E1a \\
& & 06:00 p. m. & E1b \\
E2 & En construcción & Obra gris terminada & E2a \\
E3 & En construcción & 50\% de avance * & E2b \\
\hline * & Terminado al 100\%, desocupado. & Única & E3 \\
\hline
\end{tabular}

* Obra gris terminada con un 50\% de avance en acabados y elementos no estructurales.

\section{Caso: Edificio E1}

Los resultados obtenidos para el edificio E1 en la dirección NS se muestran en la tabla 6 y la figura 4. Para este edificio se utilizó un único eje de medición, denominado eje A. Los EF y las FT se deben leer de izquierda a derecha, con el primer periodo (T1) ubicado al extremo derecho de la gráfica, el segundo periodo (T2) después del primero hacia la izquierda y así sucesivamente.

Tabla 6. Periodos naturales para edificio E1 dirección NS.

\begin{tabular}{ccccccccccc}
\hline & \multicolumn{3}{c}{ E1a-NS } & \multicolumn{5}{c}{ E1b-NS } \\
\cline { 2 - 12 } & $T 1(\mathrm{~s})$ & $T 2(\mathrm{~s})$ & $T 3(\mathrm{~s})$ & $T 4(\mathrm{~s})$ & $T 5(\mathrm{~s})$ & $T 1(\mathrm{~s})$ & $T 2(\mathrm{~s})$ & $T 3(\mathrm{~s})$ & $T 4(\mathrm{~s})$ & $T 5(\mathrm{~s})$ \\
\hline EF & 1.63 & 0.95 & 0.50 & 0.40 & 0.27 & 1.63 & 0.95 & 0.50 & 0.40 & 0.27 \\
FT & 1.62 & 0.96 & 0.49 & 0.40 & 0.27 & 1.60 & 0.96 & 0.49 & 0.40 & 0.26 \\
Dif. (s) & 0.010 & 0.002 & 0.004 & 0.001 & 0.001 & 0.031 & 0.014 & 0.002 & 0.004 & 0.004 \\
$\%$ dif. & $0.6 \%$ & $0.2 \%$ & $0.9 \%$ & $0.2 \%$ & $0.5 \%$ & $2.0 \%$ & $1.5 \%$ & $0.4 \%$ & $1.1 \%$ & $1.5 \%$ \\
\hline
\end{tabular}


Ela Espectros de Fourier NS
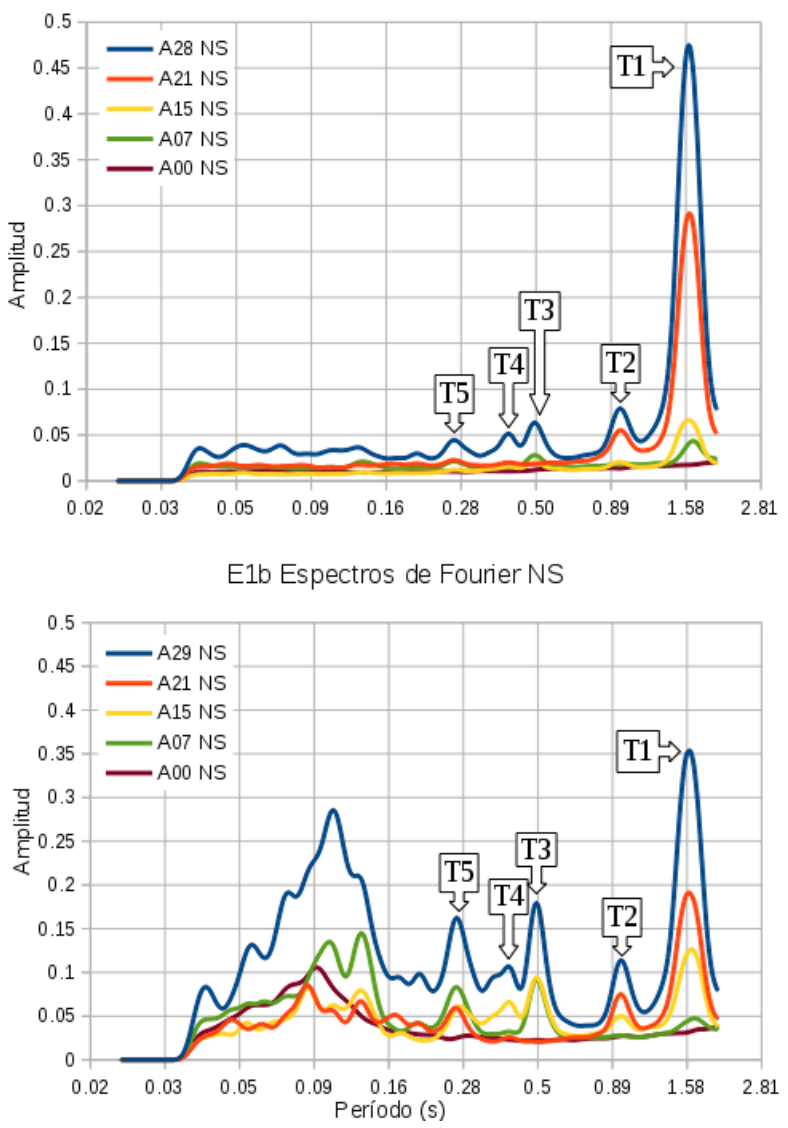

E1a Funciones de Transferencia NS

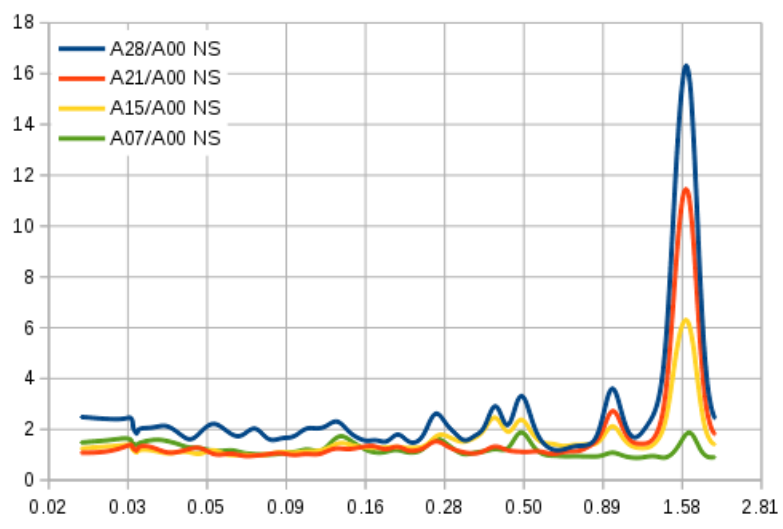

E1b Funciones de Transferencia NS

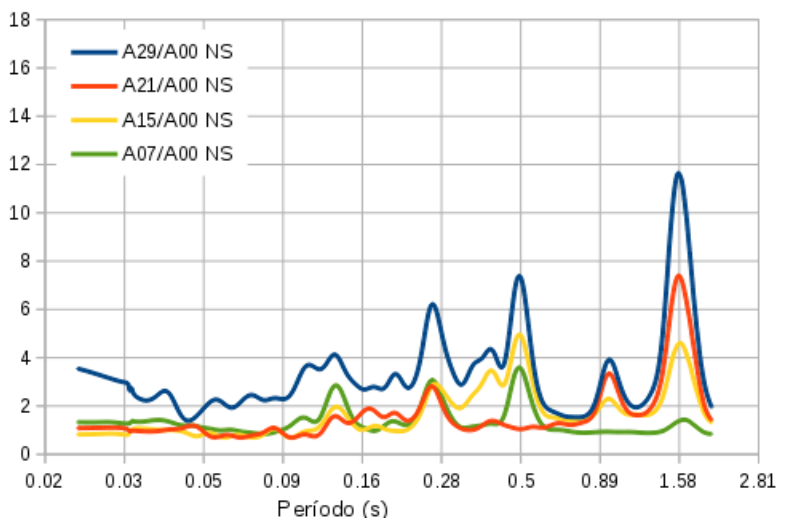

Figura 4. EF y FT para E1, dirección NS

En la figura 4, para la prueba E1a se aprecian cinco picos entre los $2.8 \mathrm{~s}$ y los $0.28 \mathrm{~s}$, tanto para los EF como para las FT, con el tercer (T3) y cuarto (T4) periodo en un "pico doble". Se puede asegurar que es un pico doble debido a que aparece en las FT, no solo en los EF. Este tipo de picos pueden indicar un posible acoplamiento entre dos modos de vibración, generalmente uno traslacional con uno torsional. Los mismos tienen una amplitud similar en el caso de la prueba realizada a las 3:00 am (E1a) pero con mayor amplitud para el $T 3$ en el caso de la prueba realizada a las 6:00 pm (E1b).

Los cinco picos entre los $2.8 \mathrm{~s}$ y $0.28 \mathrm{~s}$ corresponden a modos de vibración naturales del sistema, el edificio E1 dirección NS en este caso. Para la prueba E1b en los EF, antes de los $0.16 \mathrm{~s}$, aparecen varios picos adicionales, incluso para el caso del nivel 0 (A00 NS). Con el cálculo de las FT se logran eliminar varios de estos picos en las altas frecuencias (bajos periodos), producto probablemente de algunas vibraciones externas provenientes, por ejemplo, de máquinas u otros aparatos mecánicos que produzcan movimiento.

Se puede observar que las amplitudes espectrales (eje vertical en la figura 4) correspondientes al período fundamental (Tl) aumentan con la altura del edificio. Esto es de esperarse si se recuerda que la deformada correspondiente al modo 1 es similar a la de una viga en voladizo, por lo que los desplazamientos aumentan conforme nos alejamos de la base. Otro punto importante es que el nivel superior muestra todos los picos, a diferencia de algunos niveles en que esto no sucede. Por ejemplo, el EF de la prueba Ela para el nivel 21 muestra claramente los dos primeros períodos, no así el T3 (ver figura 4). Esto probablemente se deba a que en este nivel no hay un desplazamiento importante correspondiente al modo asociado al periodo 
T3, por lo que estos no se manifiestan en el espectro de este nivel pero sí en los espectros de otros niveles.

En la tabla 6 se muestra la diferencia entre los periodos naturales estimados por medio de los EF y FT de dos diferentes formas, diferencia total en segundos y como porcentaje, calculadas según la ecuación 1. En el caso de los porcentajes, ninguno supera el $2 \%$, lo cual es despreciable para efectos ingenieriles. A continuación, en la figura 5 y la tabla 7 se muestran los resultados para la dirección EO.

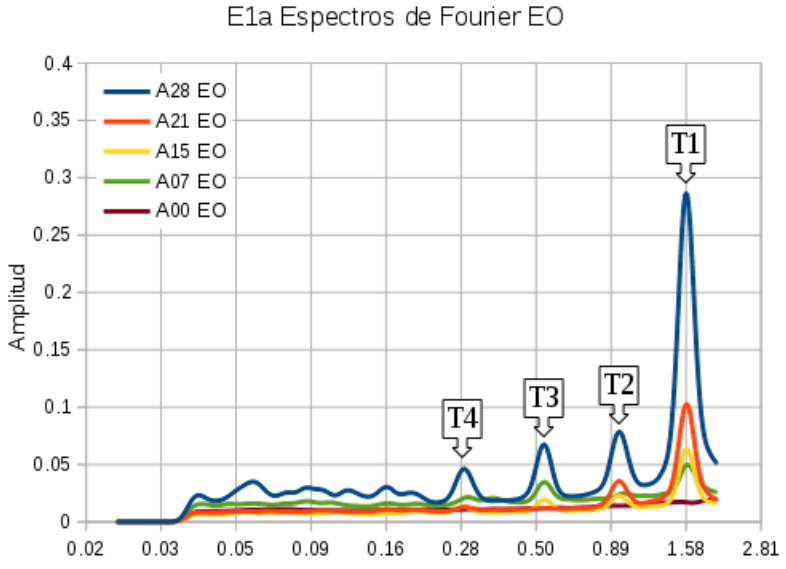

E1b Espectros de Fourier EO

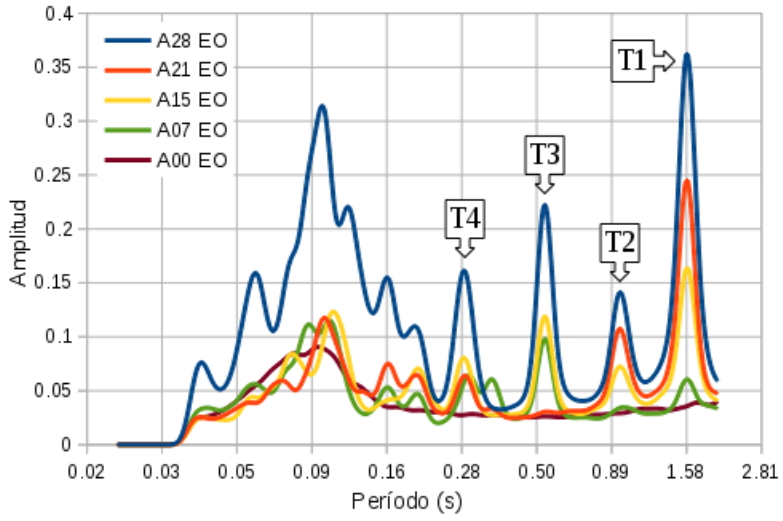

E1a Funciones de Transferencia EO

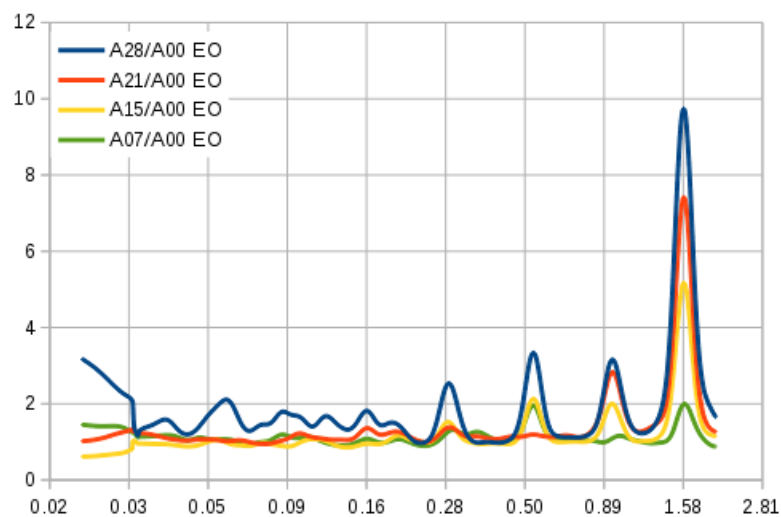

E1b Funciones de Transferencia EO

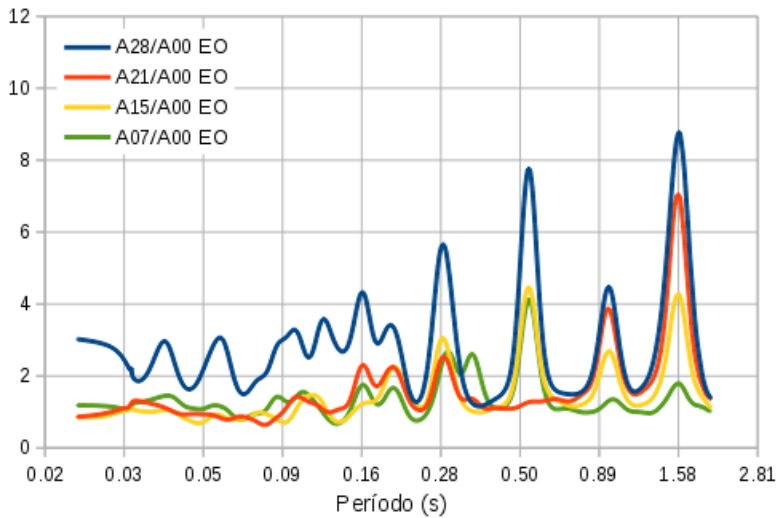

Figura 5. EF y FT para E1, dirección EO

Tabla 7. Periodos naturales para edificio E1 dirección EO.

\begin{tabular}{ccccccccc}
\hline & \multicolumn{4}{c}{ E1a-EO } & \multicolumn{4}{c}{ E1b-EO } \\
\cline { 2 - 9 } & $T 1(\mathrm{~s})$ & $T 2(\mathrm{~s})$ & $T 3(\mathrm{~s})$ & $T 4(\mathrm{~s})$ & $T 1(\mathrm{~s})$ & $T 2(\mathrm{~s})$ & $T 3(\mathrm{~s})$ & $T 4(\mathrm{~s})$ \\
\hline EF & 1.59 & 0.95 & 0.53 & 0.28 & 1.59 & 0.95 & 0.53 & 0.28 \\
FT & 1.58 & 0.96 & 0.53 & 0.29 & 1.58 & 0.96 & 0.53 & 0.29 \\
Dif. (s) & 0.012 & 0.005 & 0.001 & 0.007 & 0.012 & 0.005 & 0.001 & 0.008 \\
$\%$ dif. & $0.8 \%$ & $0.5 \%$ & $0.1 \%$ & $2.6 \%$ & $0.8 \%$ & $0.5 \%$ & $0.1 \%$ & $3.0 \%$ \\
\hline
\end{tabular}

Para este caso, se aprecian cuatro picos claramente entre $\operatorname{los} 2.8 \mathrm{~s}$ y $0.28 \mathrm{~s}$, uno menos que en la dirección NS. No hay presencia de picos dobles con la única excepción del nivel 07 para la prueba E1b, por lo que este no se presenta en la tabla 6 . Al igual que para la dirección NS, antes de los $0.16 \mathrm{~s}$ hay 
manifestaciones de las altas frecuencias para los EF de la prueba E1b, que se reducen en alguna medida con el cálculo de la FT. Así mismo, la amplitud correspondiente al periodo fundamental disminuye con la altura del edificio y algunos niveles no muestran todos los picos, como sí lo hace el nivel superior.

Para facilidad de comparación entre los periodos naturales estimados por las pruebas E1a (3:00 am) y E1b (6:00 pm) se construyó la tabla 8, siguiendo la ecuación 2 presentada en la metodología.

Tabla 8. Diferencias en periodos entre E1a y E1b.

\begin{tabular}{cccccccccc}
\hline & \multicolumn{4}{c}{ Dirección NS } & \multicolumn{4}{c}{ Dirección EO } \\
\cline { 2 - 10 } & $T 1(\mathrm{~s})$ & $T 2(\mathrm{~s})$ & $T 3(\mathrm{~s})$ & $T 4(\mathrm{~s})$ & $T 5(\mathrm{~s})$ & $T 1(\mathrm{~s})$ & $T 2(\mathrm{~s})$ & $T 3(\mathrm{~s})$ & $T 4(\mathrm{~s})$ \\
\hline EF & $0 \%$ & $0 \%$ & $0 \%$ & $2 \%$ & $-1 \%$ & $0 \%$ & $0 \%$ & $0 \%$ & $-1 \%$ \\
FT & $1 \%$ & $-1 \%$ & $-1 \%$ & $1 \%$ & $1 \%$ & $0 \%$ & $0 \%$ & $0 \%$ & $-1 \%$ \\
\hline
\end{tabular}

Como se aprecia en la tabla 8, la variación entre la estimación de los periodos en el caso de nivel de operación mínimo (E1a) y máximo (E1b) es a lo sumo igual a un $2 \%$, lo cual para efectos prácticos no representa un diferencia significativa. Con respecto a las formas de los EF y las FT se notan varias diferencias (ver figuras 4 y 5), entre ellas las amplitudes de los periodos naturales, más aún la diferencia entre el aporte del modo fundamental en el nivel de operación mínima versus operación máxima. En la prueba E1a la amplitud del modo fundamental es considerablemente superior a las de los otros modos, mientras que en la prueba E1b esta superioridad disminuye considerablemente.

Otra diferencia importante es que para el nivel de operación mínimo no se presenta participación de las altas frecuencias, apoyando la teoría de que éstas se deben a algún funcionamiento de una máquina o aparato que produce vibraciones y que se activa durante el periodo de máxima operación del edificio.

\section{Caso: Edificio E2}

A continuación se muestran los resultados obtenidos para la dirección NS. En la tabla 9 se muestran los valores de los periodos naturales y en la figura 6 se muestran los EF y las FT.

Tabla 9. Periodos naturales para edificio E2 dirección NS.

\begin{tabular}{|c|c|c|c|c|c|c|c|c|c|c|c|c|}
\hline \multirow[b]{3}{*}{ Eje } & \multicolumn{6}{|c|}{ E2a-NS } & \multicolumn{6}{|c|}{ E2b-NS } \\
\hline & \multicolumn{3}{|c|}{$T 1(\mathrm{~s})$} & \multicolumn{3}{|c|}{$T 2(\mathrm{~s})$} & \multicolumn{3}{|c|}{$T 1(\mathrm{~s})$} & \multicolumn{3}{|c|}{$T 2(\mathrm{~s})$} \\
\hline & $\mathrm{A}$ & B & $\mathrm{C}$ & A & B & $\mathrm{C}$ & A & B & $\mathrm{C}$ & A & B & $\mathrm{C}$ \\
\hline $\mathrm{EF}$ & 1.05 & 1.10 & 1.09 & 0.29 & 0.30 & 0.30 & 1.02 & 1.06 & 1.06 & 0.29 & 0.29 & 0.29 \\
\hline FT & 1.04 & 1.12 & 1.16 & 0.29 & 0.30 & 0.29 & 1.02 & 1.06 & 1.05 & 0.29 & 0.29 & 0.29 \\
\hline Tprom EF & & $1.08 \mathrm{~s}$ & & & $0.29 \mathrm{~s}$ & & & $1.05 \mathrm{~s}$ & & & $0.29 \mathrm{~s}$ & \\
\hline Tprom FT & & $1.10 \mathrm{~s}$ & & & $0.29 \mathrm{~s}$ & & & $1.04 \mathrm{~s}$ & & & $0.29 \mathrm{~s}$ & \\
\hline Dif. (s) & & $0.026 \mathrm{~s}$ & & & $0.002 \mathrm{~s}$ & & & $0.003 \mathrm{~s}$ & & & $0.002 \mathrm{~s}$ & \\
\hline$\%$ dif. & & $2.4 \%$ & & & $0.7 \%$ & & & $0.3 \%$ & & & $0.7 \%$ & \\
\hline
\end{tabular}


E2a Espectros de Fourier NS

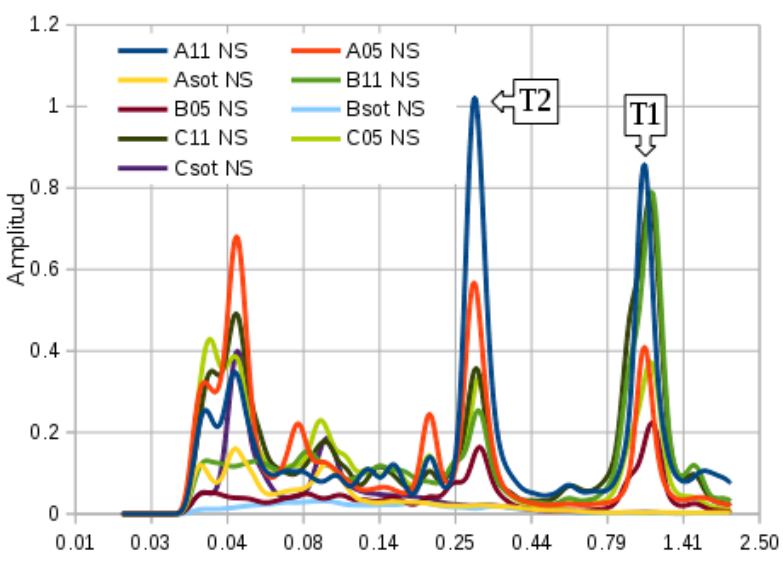

E2b Espectros de Fourier NS

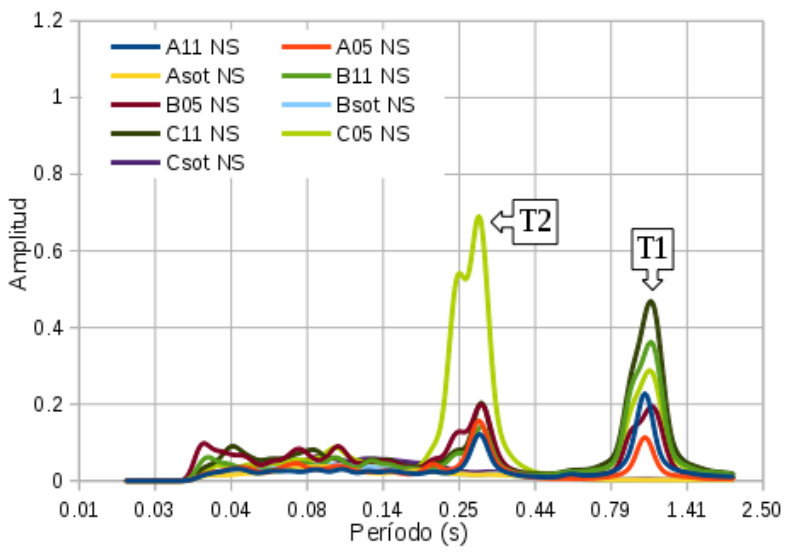

E2a Funciones de Transferencia NS

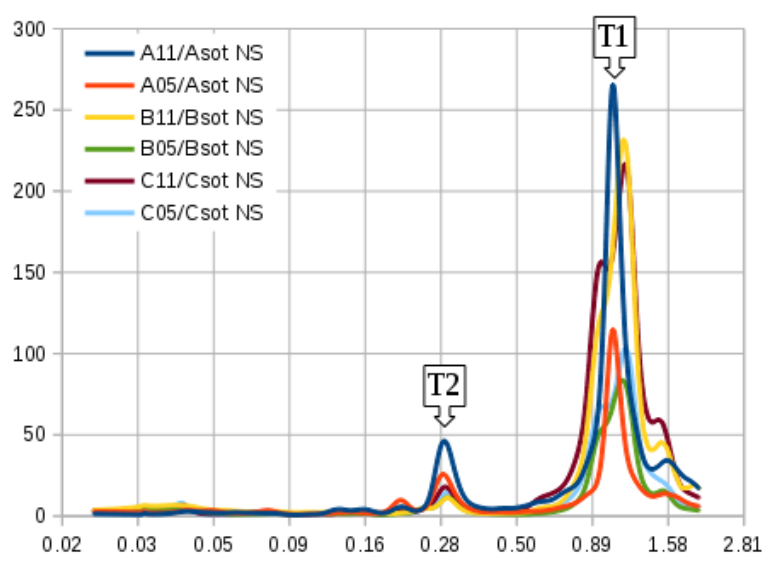

E2b Funciones de Transferencia NS

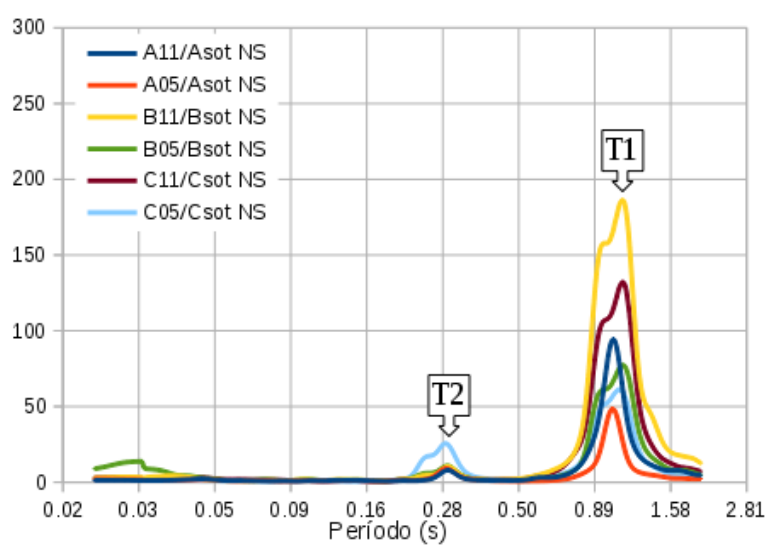

Figura 6. EF y FT para E2, dirección NS

Para el caso del E2, se utilizaron tres ejes de medición (ejes A, B, C) a diferencia de un único eje de medición utilizado para el E1 (eje A). Por esta razón es necesario calcular los periodos naturales promedio (Tprom) para cada uno de los EF y FT.

En la dirección NS, tanto de los EF como de las FT se pudieron determinar dos periodos naturales denominados $T 1$ y $T 2$. La estimación de estos para cada eje es consistente entre sí, por lo que los resultados obtenidos por cada eje se consideran válidos y se utilizan para obtener los valores de periodos promedio. No hay presencia de picos dobles, sin embargo la alineación de los picos para $T 1$ entre ejes no es absoluta. Esto es de esperar si se recuerda que los periodos estimados para los EF y FT por cada eje son en realidad un promedio. En las FT mostradas en la figura 6, por ejemplo el caso de B11/Bsot, se aprecia que la base cerca del pico correspondiente a $T 1$ (cerca de la amplitud 140) es más ancha que la de A05/Asot (cerca de la amplitud 40). Esto podría deberse a un acoplamiento de modos, traslacional y torsional, que se encuentran tan cercanos que no pueden diferenciarse con claridad.

En el caso de la prueba E2a (estructura en obra gris), en los EF se muestra una importante participación de frecuencias altas antes de los $0.2 \mathrm{~s}$, las cuales disminuyen considerablemente en amplitud en el cálculo de las FT. Cabe recalcar que durante la ejecución de la prueba E2a, se estaban llevando a cabo 
actividades de construcción propias de la instalación de elementos no estructurales, las cuales involucran el uso de maquinaría liviana que pudo haber sido la responsable de estas altas frecuencias. Al igual que en el edificio E1, las amplitudes correspondientes al periodo $T 1$ aumentan con la altura del edificio, aunque en este caso la amplitud del periodo $T 2$ es mayor que la del $T 1$ para los EF, no así para las FT. Todos los niveles, excepto el sótano obviamente, presentan los picos correspondientes a los dos periodos, algo que no ocurrió en el caso del edificio E1. Las diferencias entre los periodos naturales promedio estimados por medio de EF y FT son bajas nuevamente, con un máximo del $2.4 \%$, lo que para efectos prácticos es insignificante.

Seguidamente se muestran los resultados en la dirección EO, los periodos naturales en la Tabla 10, y los EF y FT en la figura 7.

Tabla 10. Periodos naturales para edificio E2 dirección EO.

\begin{tabular}{ccccccccccccc}
\hline & \multicolumn{1}{c}{ E2a-EO } & \multicolumn{1}{c}{ E2b-EO } \\
\cline { 2 - 14 } & $T 1-\mathrm{C}(\mathrm{s})$ & \multicolumn{1}{c}{$T 1(\mathrm{~s})$} & $T 2-\mathrm{C}(\mathrm{s})$ & \multicolumn{1}{c}{$T 2(\mathrm{~s})$} & $T 1-\mathrm{C}(\mathrm{s})$ & $T 1(\mathrm{~s})$ & $T 2-\mathrm{C}(\mathrm{s})$ & $T 2(\mathrm{~s})$ \\
\hline Eje & $\mathrm{C}$ & $\mathrm{A}$ & $\mathrm{B}$ & $\mathrm{C}$ & $\mathrm{A}$ & $\mathrm{B}$ & $\mathrm{C}$ & $\mathrm{A}$ & $\mathrm{B}$ & $\mathrm{C}$ & $\mathrm{A}$ & $\mathrm{B}$ \\
\hline EF & 1.12 & 0.94 & 0.94 & 0.29 & 0.25 & 0.25 & 1.12 & 0.93 & 0.92 & 0.30 & 0.24 & 0.24 \\
FT & 1.14 & 0.94 & 0.93 & 0.29 & 0.24 & 0.25 & 1.12 & 0.93 & 0.92 & 0.29 & 0.24 & 0.24 \\
Tprom EF & $1.12 \mathrm{~s}$ & $0.94 \mathrm{~s}$ & $0.29 \mathrm{~s}$ & $0.25 \mathrm{~s}$ & $1.12 \mathrm{~s}$ & $0.92 \mathrm{~s}$ & $0.30 \mathrm{~s}$ & $0.25 \mathrm{~s}$ \\
Tprom FT & $1.14 \mathrm{~s}$ & $0.94 \mathrm{~s}$ & $0.29 \mathrm{~s}$ & $0.24 \mathrm{~s}$ & $1.12 \mathrm{~s}$ & $0.92 \mathrm{~s}$ & $0.29 \mathrm{~s}$ & $0.24 \mathrm{~s}$ \\
Dif. (s) & 0.022 & 0.004 & 0.001 & 0.003 & 0.001 & 0.002 & 0.007 & 0.004 \\
\% dif. & $1.9 \%$ & $0.4 \%$ & $0.2 \%$ & $1.1 \%$ & $0.1 \%$ & $0.3 \%$ & $2.6 \%$ & $1.5 \%$ \\
\hline
\end{tabular}

La primera diferencia que salta a la vista entre las Tabla 10 y 9 es la división de cada periodo en dos partes, Tn-C y Tn, con $n$ igual a 1 y 2 . Esto se debe a que en esta dirección el periodo medido por el eje $\mathrm{C}$ no concordaba con los medidos en los ejes A y B. Al examinar las FT de la figura 7 alrededor de los periodos 0.9 s y $0.3 \mathrm{~s}$, se comprueba que los picos entre los ejes A y B se alinean mutuamente, no así los del eje C, conteniendo en este caso diferencias importantes del orden del $22 \%$ al $16 \%$ con respecto a los otros ejes.

La razón de esto puede deberse a la irregularidad en planta que presenta este edificio. Si recordamos la geometría de la planta típica de la figura 2, podemos ver que tiene forma de "C". Lo anterior propicia que unas secciones de la estructura se comporten diferente al resto del edificio, las cuales se nombran como alas en este caso y se muestran en la figura 8. 
E2a Espectros de Fourier EO

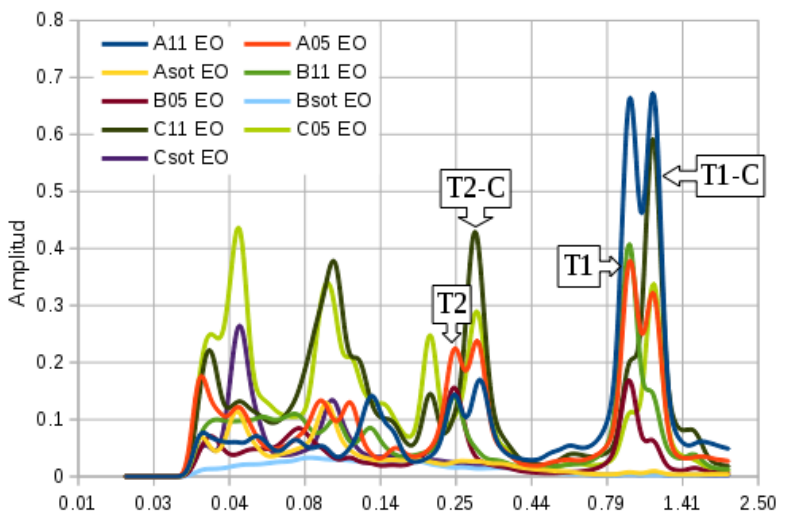

E2b Espectros de Fourier EO

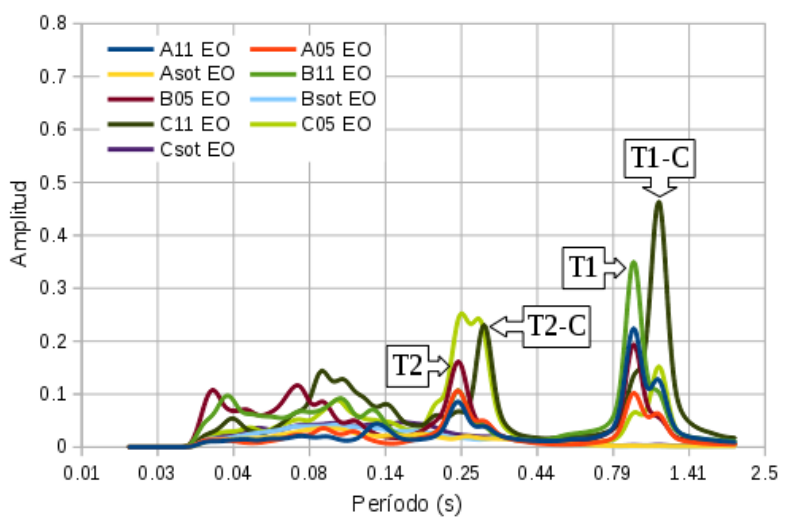

E2a Funciones de Transferencia EO

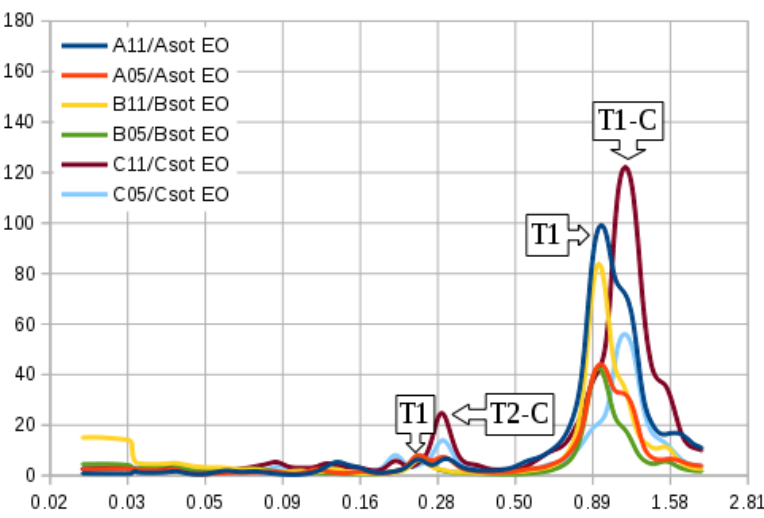

E2b Funciones de Transferencia EO

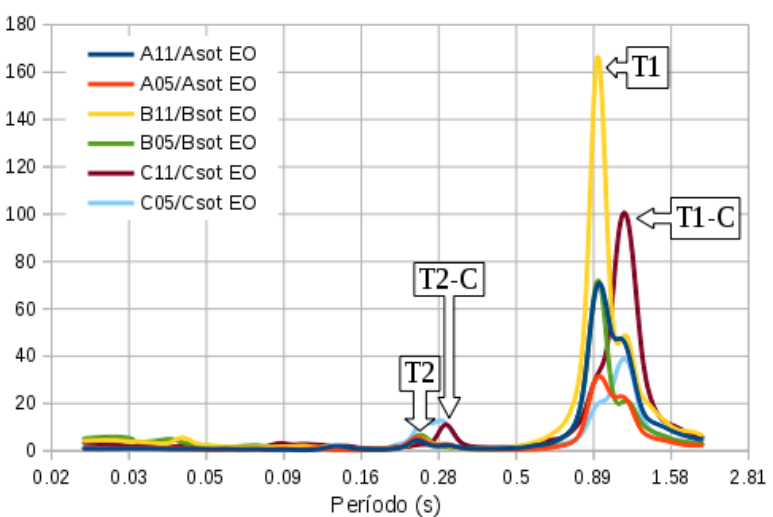

Figura 7. EF y FT para E2, dirección EO

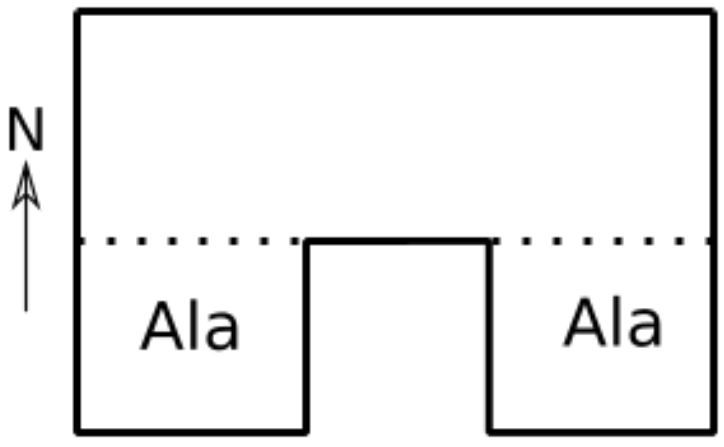

Figura 8. Secciones que se comportan como alas

Como se aprecia en la figura 9, en la dirección NS el edificio se desplaza como un solo cuerpo, por lo que los periodos naturales entre todos los ejes coinciden, mientras que en la dirección EO se tiene el comportamiento diferente. Este consiste en un movimiento del edificio como un solo cuerpo hasta cierto punto y luego de esto las alas siguen desplazándose en esta dirección, lo que se refleja en la curvatura de la deformada de estas. La hipótesis de que las alas continúan desplazándose más allá de lo que lo hace el cuerpo rígido se sustenta en que el periodo natural del eje ubicado en ellas es mayor al eje del centro edificio, es decir, las alas son más flexibles y tardan más tiempo en realizar un ciclo completo de movimiento 
oscilatorio. En la figura 2 podemos ver que el eje $\mathrm{C}$ se encuentra en una de las alas, lo que explicaría que el periodo de este sea diferente y más largo que para los ejes A y B.
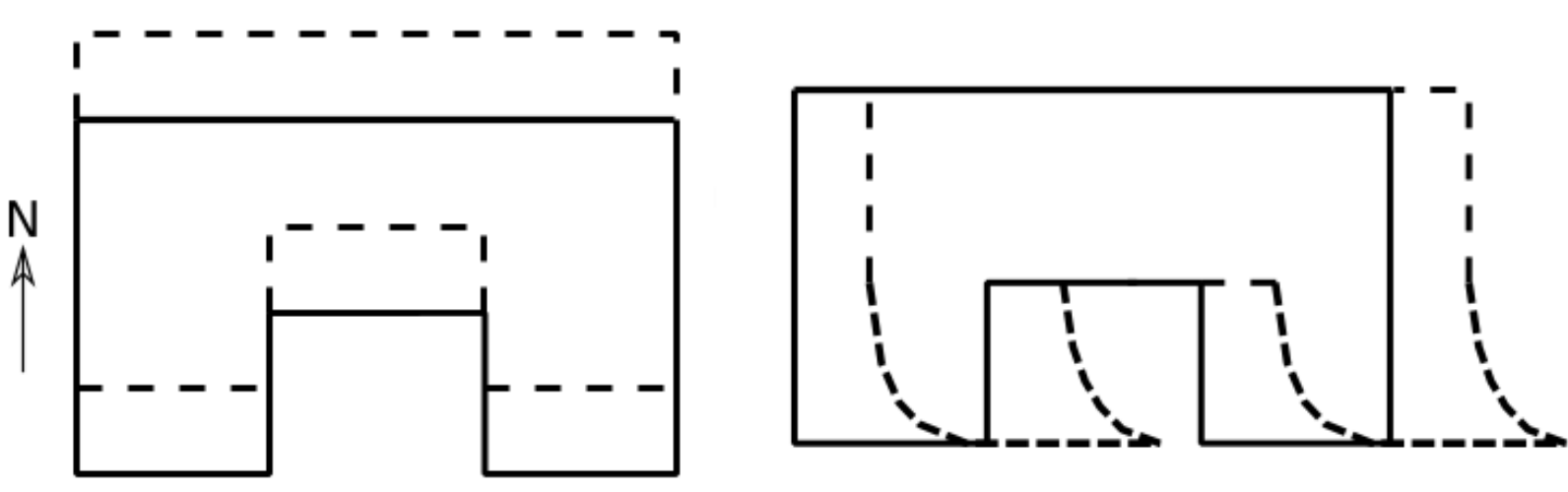

Figura 9. Desplazamiento de E2 en sus direcciones principales

En el caso de la prueba E2a, para el EF se tienen picos dobles que se eliminan a la hora de estimar las FT. Por esta razón es que para este caso se tuvo la necesidad de recurrir a las FT con el fin de lograr una identificación más clara de los periodos naturales. Al igual que en la dirección NS, en los EF existe una presencia importante de altas frecuencias antes de los $0.2 \mathrm{~s}$ que se elimina en el cálculo de las FT. Para el periodo fundamental las amplitudes aumentan con la altura del edifico, los dos periodos se manifiestan en todos los niveles y las diferencias entre los valores estimados de periodos naturales por los EF y FT son mínimas, igual a $2.6 \%$ en el peor de los casos, lo cual no es una diferencia significativa.

En la tabla 11 se muestran las diferencias entre la prueba E2a (estructura en obra gris) y E2b (obra gris más un avance del $50 \%$ en acabados y elementos no estructurales), para facilidad de comparación, calculadas según la ecuación 2.

Tabla 11. Diferencias en periodos entre E2a y E2b.

\begin{tabular}{|c|c|c|c|c|c|c|c|c|c|c|c|c|}
\hline & & & irecc & ión $\mathrm{N}$ & & & & & recci & lón EO & & \\
\hline & & $T 1(\mathrm{~s}$ & & & $T 2(\mathrm{~s})$ & & $T 1-\mathrm{C}(\mathrm{s})$ & $T 1$ & & $T 2-\mathrm{C}(\mathrm{s})$ & $T 2$ & \\
\hline Eje & A & B & $\mathrm{C}$ & A & B & $\mathrm{C}$ & $\mathrm{C}$ & A & B & $\mathrm{C}$ & A & B \\
\hline $\mathrm{EF}$ & $2 \%$ & $4 \%$ & $3 \%$ & $-1 \%$ & $2 \%$ & $1 \%$ & $0 \%$ & $2 \%$ & $1 \%$ & $-3 \%$ & $2 \%$ & $1 \%$ \\
\hline FT & $2 \%$ & $6 \%$ & $10 \%$ & $-1 \%$ & $2 \%$ & $2 \%$ & $2 \%$ & $2 \%$ & $1 \%$ & $0 \%$ & $2 \%$ & $0 \%$ \\
\hline
\end{tabular}

Para la dirección NS, la variación en periodo es más significativa para las FT que para los EF, con un máximo de $4 \%$ y $10 \%$ respectivamente. De igual forma, el periodo más afectado es el $T 1$, ya que el $T 2$ presenta cambios del orden del $2 \%$ solamente. Las diferencias en los periodos entre las dos mediciones para la dirección EO son aún menores que para la dirección NS, con un máximo de 3\%, y nuevamente la mayoría de los periodos para la prueba E2a son mayores que los de E2b.

Con excepción del T2 para el eje A en dirección NS y del T2-C en la dirección EO, los periodos correspondientes a la E2a son mayores que los de E2b, lo cual podría indicar que el aporte en rigidez de los acabados y elementos no estructurales superó el efecto del aumento en masa de la estructura proporcionado por los mismos. Lo anterior es consistente con lo concluido por Cantieni y Biro (2005), explicado por medio de la ecuación 3 , donde se evidencia que a mayor rigidez, menor periodo y a mayor masa, mayor periodo. 


$$
T_{n}=2 \pi \sqrt{\frac{m}{k}}
$$

donde $T_{n}$ : periodo natural, $m$ es la masa participativa de la estructura, y $k$ es la rigidez de la estructura.

Con respecto al amortiguamiento $(\beta)$, se analizaron los registros correspondientes a todos los ejes en la dirección NS y a los ejes A y B para la dirección EO, esto debido a que el eje $\mathrm{C}$ se encuentra en la sección del edificio que se comporta como ala. Como se explicó anteriormente en la metodología, el criterio de validación del amortiguamiento fue el comparar el periodo natural estimado por medio de la técnica de decaimiento aleatorio con el estimado por medio de los EF y FT.

Se realizaron varias estimaciones del amortiguamiento y se observó que es sumamente sensible a la estimación del periodo natural. Esto implica que para diferencias pequeñas entre el periodo natural obtenido por los EF y FT versus el obtenido por la técnica de decaimiento aleatorio, el cambio en el amortiguamiento fue significativo. Por esta razón se tomó como límite superior una diferencia igual a 1.5\% entre los periodos naturales para considerar como válido el registro para el cálculo del amortiguamiento. Otro criterio es el del número de ventanas promediadas utilizadas en la técnica, las cuales según Chang (1975) deberían ser del orden de 2000. En la tabla 12 se muestran los registros utilizados para la estimación de los amortiguamientos, el número de ventanas se trató de mantener lo más cerca posible a 2000.

Tabla 12. Amortiguamientos por registro.

\begin{tabular}{ccccccccc}
\hline & \multicolumn{4}{c}{ E2a } & \multicolumn{5}{c}{ E2b } \\
\hline Registros & A11 NS & A05 NS & B11 EO & B05 EO & A11 NS & A05 NS & B11 EO & B05 EO \\
\hline$\beta(\%)$ & 0.52 & 0.57 & 0.41 & 0.41 & 0.97 & 0.99 & 0.54 & 0.55 \\
Dif. entre $T 1(\%)$ & 0.6 & 0.5 & 0.6 & 0.6 & 0.2 & 0.2 & 0.3 & 0.3 \\
Ventanas & 1508 & 1506 & 1876 & 1874 & 2003 & 2002 & 1675 & 1674 \\
\hline
\end{tabular}

Las diferencias entre los periodos naturales (Dif. entre $T 1$ ) son menores que $0.6 \%$ en todos los casos. Para cada una de las pruebas realizadas se utilizaron cuatro registros. Los acelerogramas del eje C se tomaron en cuenta sólo para la dirección NS, donde el edificio se comporta como un solo cuerpo, sin embargo estos no cumplieron con los criterios de selección. En la tabla 13 se muestran los amortiguamientos promedio obtenidos en cada prueba para cada dirección. También se expone la diferencia entre los periodos de cada prueba (ecuación 4), así como cuánto representa el aumento con respecto a la prueba E2a (ecuación $5)$.

Tabla 13. Amortiguamientos promedio.

\begin{tabular}{ccccc}
\hline & \multicolumn{2}{c}{$\beta$} & \multicolumn{2}{c}{ Diferencias } \\
\cline { 2 - 5 } Dirección & E2a & E2b & Aumento & Representa \\
\hline NS & $0.54 \%$ & $0.98 \%$ & $0.44 \%$ & $82 \%$ \\
EO & $0.41 \%$ & $0.55 \%$ & $0.13 \%$ & $32 \%$ \\
\hline
\end{tabular}

Aumento $=\beta E 2 b-\beta E 2 a$

Representa $=\frac{\beta E 2 b-\beta E 2 a}{\beta E 2 a}$ 
donde $\beta E 2 a$ es el amortiguamiento calculado para la prueba E2a, y $\beta E 2 b$ es el amortiguamiento calculado para la prueba E2b.

Se puede ver que los amortiguamientos estimados para ambas pruebas no superan el $1 \%$, pero se debe recordar que al momento de la prueba E2a el edificio se encontraba en obra gris, sin ningún tipo de acabado o elemento no estructural y sujeto a amplitudes mínimas de aceleración, ciertamente en el rango elástico del edificio. Para la prueba E2b, se tenía un 50\% de avance en acabados y elementos no estructurales, los cuales en su mayoría correspondían a fachadas, acabados, pisos, divisiones y tuberías en zonas comunes. El edificio será destinado para oficinas y comercio, cada espacio se entrega en obra gris para que el cliente lo acondicione a su gusto, lo que implica que no había ningún tipo de acabados, pisos, muebles o divisiones livianas en todas las zonas para alquilar o vender, que son la mayoría del área de construcción.

En cuanto a las diferencias, se percibe un aumento del amortiguamiento en ambas direcciones siendo mayor para el caso de la dirección NS. Era de esperarse que el amortiguamiento fuera mayor para la prueba E2b debido al $50 \%$ de avance en acabados y elementos no estructurales.

\section{Caso: Edificio E3}

A continuación, en la figura 10 y la tabla 14, se muestran los resultados obtenidos para ambas direcciones del edificio E3.
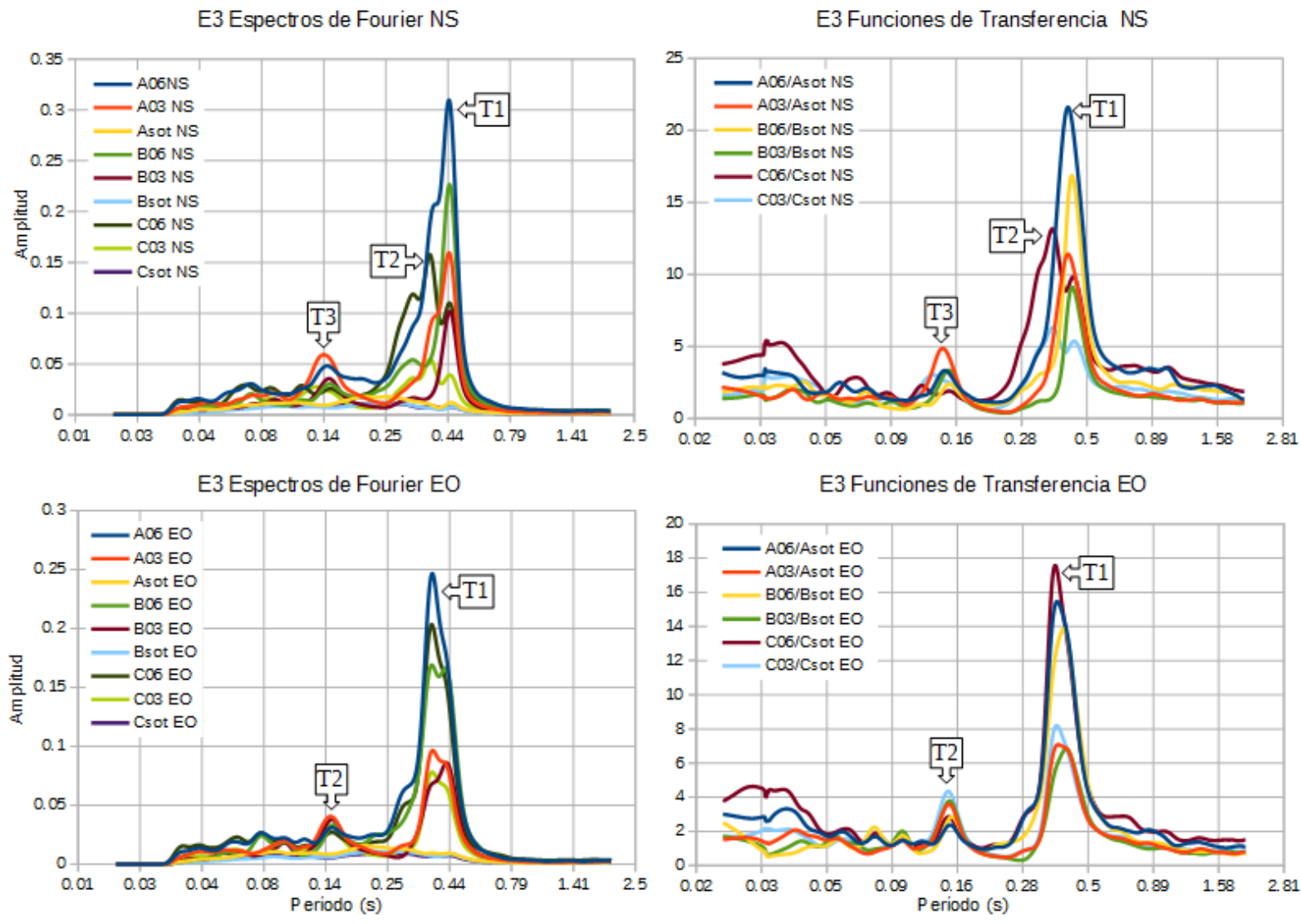

E3 Funciones de Transferencia EO

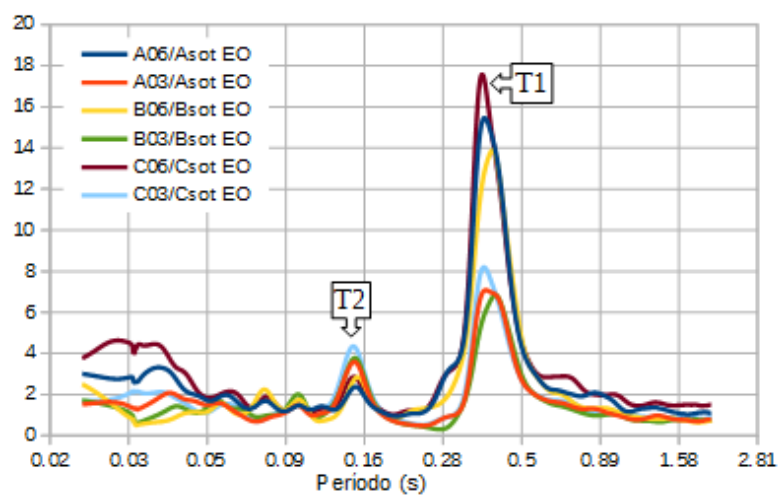

Figura 10. EF y FT para E3 
Tabla 14. Periodos naturales para edificio E3.

\begin{tabular}{|c|c|c|c|c|c|c|c|c|c|c|c|c|c|}
\hline \multirow[b]{3}{*}{ Eje } & \multicolumn{7}{|c|}{ E3-NS } & \multicolumn{6}{|c|}{ E3-EO } \\
\hline & \multicolumn{3}{|c|}{$T 1(\mathrm{~s})$} & \multirow{2}{*}{$\frac{T 2(\mathrm{~s})}{\mathrm{C}}$} & \multicolumn{3}{|c|}{$T 3(\mathrm{~s})$} & \multicolumn{3}{|c|}{$T 1(\mathrm{~s})$} & \multicolumn{3}{|c|}{$T 2(\mathrm{~s})$} \\
\hline & $\mathrm{A}$ & B & $\mathrm{C}$ & & $\mathrm{A}$ & B & $\mathrm{C}$ & A & B & $\mathrm{C}$ & A & B & $\mathrm{C}$ \\
\hline $\mathrm{EF}$ & 0.45 & 0.45 & 0.46 & 0.38 & 0.14 & 0.15 & 0.15 & 0.39 & 0.38 & 0.38 & 0.15 & 0.15 & 0.15 \\
\hline FT & 0.42 & 0.44 & 0.45 & 0.37 & 0.14 & 0.15 & 0.15 & 0.39 & 0.41 & 0.38 & 0.15 & 0.15 & 0.15 \\
\hline T prom $\mathrm{EF}$ & & $0.45 \mathrm{~s}$ & & 0.38 & & $0.15 \mathrm{~s}$ & & & $0.38 \mathrm{~s}$ & & & $0.15 \mathrm{~s}$ & \\
\hline T prom $\mathrm{FT}$ & & $0.44 \mathrm{~s}$ & & 0.37 & & $0.15 \mathrm{~s}$ & & & $0.39 \mathrm{~s}$ & & & $0.15 \mathrm{~s}$ & \\
\hline Dif. (s) & & 0.017 & & 0.01 & & 0.001 & & & 0.007 & & & 0.001 & \\
\hline$\%$ dif. & & $3.8 \%$ & & $2.7 \%$ & & $0.6 \%$ & & & $1.9 \%$ & & & $0.9 \%$ & \\
\hline
\end{tabular}

En la figura 10 se puede observar que existen dos grupos de picos: el primero alrededor de los $0.45 \mathrm{~s}$ y el segundo alrededor de los $0.15 \mathrm{~s}$. En los EF C06 NS, C03 NS y B06 EO hay presencia de picos dobles, por lo que se debió recurrir a las FT para aclarar este tema.

En el caso de la dirección EO los picos dobles se eliminan al calcular las FT, porque los picos quedan alineados con una diferencia máxima del $8 \%$ entre los periodos estimados por los ejes C y B. Esta diferencia se consideró aceptable, recordando que los valores de estos periodos son un promedio. Para la dirección NS los picos persisten incluso en las FT, lo que indican que posiblemente ambos representen periodos naturales de la estructura. Por esta razón, se incluye en la tabla 14 el valor de $T 2$ estimado exclusivamente a partir de los registros del eje $\mathrm{C}$, correspondiente a los $0.38 \mathrm{~s}$ y $0.37 \mathrm{~s}$ para los EF y FT, respectivamente.

Para ambas direcciones y en todos los casos, los picos correspondientes al segundo grupo, alrededor de los $0.15 \mathrm{~s}$, se encuentran alineados y coinciden para todos los ejes. No hay presencia importante de frecuencias altas en ninguno de los EF, lo cual es consistente con el ambiente que se tuvo a la hora de realizar la MVA (bajo nivel de ruido). El edificio estaba terminado al $100 \%$ y entregado, pero todavía no estaba ocupado, por lo que no habían usuarios en la estructura, no estaba amueblado, no habían artefactos en funcionamiento como por ejemplo máquinas de aire acondicionado, generadores u otros, es decir la carga temporal se podía estimar como nula y las vibraciones operacionales como mínimas.

La amplitud del periodo fundamental aumenta con la altura del edificio y los periodos se manifiestan en todos los niveles. La mayor diferencia entre las estimaciones por EF y FT de los periodos naturales es del $3.8 \%$, la cual no es significativa.

Para este edificio se construyó un modelo matemático con el programa ETABS, donde se modeló el sistema estructural compuesto por columnas y vigas como elementos tipo marco (frame en ETABS) y los muros como elementos tipo área (shell en ETABS) con comportamiento de membrana (deformaciones exclusivamente en el plano del elemento, sin deformaciones fuera del plano). Los entrepisos se modelaron como elementos de área sin peso ni masa, con un diafragma rígido asignado a cada nodo del modelo en cada nivel. El objetivo fue calibrar el modelo utilizando la medición de vibraciones ambientales (MVA) realizada, se tomó como parámetro el periodo fundamental, es decir, que el periodo medido por medio de la prueba concordara lo más posible con el estimado por el modelo matemático.

Se tuvieron que tomar varias consideraciones para realizar el modelo y calibrarlo, las cuales usualmente no se toman en cuenta para la elaboración de los modelos que se utilizan con fines de diseño. La primera de ellas fue que las cargas impuestas al modelo deben ser lo más cercanas a las cargas existentes 
al momento de realizar la prueba, no las cargas máximas esperadas a lo largo de la vida útil del inmueble, las cuales se utilizan para su diseño estructural. Como ya se mencionó anteriormente, cuando se realizó la prueba el edificio se encontraba terminado al $100 \%$ y desocupado en su totalidad, por lo que la carga temporal se estimó como nula, tanto para entrepisos como para techos.

Otra de las consideraciones importantes a la hora de calibrar el modelo fue considerar todos los elementos estructurales como no agrietados, que en ETABS se traducen en factores de inercia iguales a la unidad. Esto debido a que desde la finalización de la construcción del edificio, no había ocurrido algún sismo considerable que pudiera agrietar los elementos, algo que no ocurre en edificios más antiguos que han soportado sismos fuertes (Murià et al., 2000).

La última consideración y de mayor efecto fue la de asignar una rigidez infinita y restringir el grado de libertad asociado a la rotación en todas las conexiones viga-columna de todos los marcos, es decir, cada conexión es infinitamente rígida y tiene libertad de movimiento vertical y horizontal, pero no de rotación. Esta se basa en que las amplitudes de las aceleraciones medidas en la MVA son muy bajas, por lo que inducen desplazamientos mínimos en la estructura, los cuales no podrían vencer las fuerzas de fricción internas de las conexiones, por lo que estas no rotarían.

El modelo generado tomando en cuenta las consideraciones anteriores estimó el periodo fundamental en $0.47 \mathrm{~s}$. En el caso de la MVA realizada, el periodo fundamental promedio para los EF fue de $0.45 \mathrm{~s}$ y el de las FT de $0.44 \mathrm{~s}$, lo que implica que el periodo calculado por el modelo tiene un error igual a $4.4 \%$ con respecto a los EF e igual a $6.8 \%$ para las FT.

\section{CONCLUSIONES}

Las diferencias entre los periodos naturales obtenidos por medio de los espectros de Fourier (EF) y las funciones de transferencia (FT) para tres edificios de concreto reforzado de 28,11 y 6 pisos, con sistemas sismo-resistente tipo dual, fueron igual a $3 \%, 2.6 \%$ y $3.8 \%$, respectivamente. Las mismas son consideradas como despreciables, más aún si se toma en cuenta que los periodos naturales son determinados por medios probabilísticos. Por esto se concluye que para los casos estudiados, se logró una determinación consistente de los periodos naturales con cualquiera de las dos técnicas (EF y FT).

Sin embargo, en los casos de picos dobles se debe recurrir a la FT para asegurarse que estos corresponden a modos de vibración de la estructura y no a alguna frecuencia de un agente externo a la prueba, por ejemplo al funcionamiento de alguna máquina. Esto debido a que, teóricamente, las FT eliminan la participación de las fuentes excitadoras, por lo que siempre será más confiable que los EF, pero requieren de un mayor esfuerzo de cálculo. Prueba de esto es que en los edificios de 28 y 6 pisos, en la dirección Norte-Sur, no se eliminan los picos dobles en las FT implicando que ambos corresponden a periodos de la estructura, mientras que para el edificio de 11 pisos en la dirección Este-Oeste sí se eliminan. Lo anterior debido a que el cálculo de las FT implica la división de dos espectros que contienen energía en las mismas frecuencias.

Tanto para los EF como para las FT, todos los periodos se manifestaron de manera clara en el nivel superior. Esto implica que para los casos estudiados bastaría tener dos puntos de medición sincronizados en el tiempo (por medio de GPS o interconectados entre sí), uno en el nivel inferior y otro en el superior para una identificación exitosa de al menos los primeros cuatro periodos naturales. 


\section{Caso: Edificio de 28 pisos (E1)}

En la dirección Norte-Sur se pudieron leer cinco periodos naturales, mientras que en la dirección Este-Oeste se identificaron cuatro. En el caso de los EF para la prueba correspondiente al nivel de operación máxima del edificio (prueba E1b, realizada a las 6:00 pm), se encontró una presencia importante de frecuencias altas, las cuales disminuyeron significativamente con el cálculo de las FT. Aun así, los EF correspondientes a la prueba de nivel de operación mínima del edificio (prueba E1a, realizada a las 3:00 am) presentaban menor manifestación de estas altas frecuencias. Se concluye que para efectos de identificación de los primeros nueve periodos naturales, el nivel de operación del edificio no influyó o modificó estos parámetros dinámicos, ya que el cambio máximo de una prueba a otra fue del $2 \%$. La diferencia se presentó para frecuencias altas $(0.2 \mathrm{~Hz}$ o $5 \mathrm{~s})$, donde la participación de estas aumentó de manera considerable para el nivel de operación máxima.

En la dirección Norte-Sur se presentaron dos periodos muy cercanos entre sí, representados en los espectros como un pico doble, correspondientes al tercer y cuarto modo de vibración ( $T 3$ y $T 4$ ). Este podría corresponder a un acoplamiento modal entre un modo traslacional y otro torsional, ya que los primeros periodos naturales correspondían a modos traslacionales, lo que es de esperar en un edificio regular. Para confirmar esta hipótesis habría que realizar una medición para la identificación del modo torsional.

\section{Caso: Edificio de 11 pisos (E2)}

Se pudieron identificar dos periodos naturales en cada una de las direcciones, con la salvedad de que para la dirección Este-Oeste se identificaron dos periodos adicionales exclusivos de una sección (ala) del edificio. Las frecuencias altas observadas en los EF se redujeron por completo en el cálculo de las FT. Las diferencias entre los periodos naturales para dos etapas de construcción diferentes, en obra gris (E2a) y con un 50\% de avance en acabados y elementos no estructurales (E2b), varían de forma importante según la dirección analizada. En el caso de la dirección Norte-Sur, la variación máxima del primer periodo (T1) es del 10\%, mientras que para el segundo periodo (T2) baja a un 2\%. En el caso de la dirección Este-Oeste, la variación es baja, con un máximo del $3 \%$. Además la mayoría de los periodos son más extensos para la prueba E2a, lo que podría indicar que el aporte en rigidez de los acabados y elementos no estructurales superó el efecto del aumento en masa de la estructura proporcionado por los mismos.

Con respecto al amortiguamiento, su valor absoluto para las dos pruebas en ambas direcciones no superó el 1\%. Hay que recordar que en ambas pruebas el edificio no se encontraba $100 \%$ terminado, no contenía las cargas temporales correspondientes a ocupación plena y las aceleraciones registradas son de amplitudes muy bajas, ciertamente correspondientes a un comportamiento elástico de la estructura. Se registró un aumento del $82 \%$ para el amortiguamiento correspondiente a la dirección Norte-Sur, contra un aumento del $32 \%$ para la dirección Este-Oeste.

Se concluye que la variación en los periodos naturales es diferente para los dos modos identificados en ambas direcciones, teniendo una variación significativa únicamente para el periodo $T 1$ en la dirección Norte-Sur. Lo anterior es consistente con la variación observada para el amortiguamiento, más significativa también en la dirección Norte-Sur.

En la FT, dirección Norte-Sur prueba E2b (ver figura 6), se apreciaron varios picos correspondientes al primer periodo (T1) con una especie de base ancha (por ejemplo, FT de B11/Bsot en la amplitud 140), que a partir de un punto se vuelve angosta en uno de sus extremos (FT de B11/Bsot en la amplitud 160) y termina en el pico asociado con el periodo $T 1$. En la parte ancha se podría estar reflejando la participación de un modo adicional, el estaría acoplado al modo T1. Por la planta irregular que presenta este edificio se 
podría estar tratando de un modo torsional acoplado a uno traslacional.

\section{Caso: Edificio de 6 pisos (E3)}

Se pudieron identificar cinco periodos naturales en total, tres para la dirección Norte-Sur y dos para la dirección Este-Oeste. En el caso de la dirección Norte-Sur, el periodo T2 solo se manifestó en el eje C, en una de las esquinas del edificio más alejadas del centro de rigidez, ya que el único ducto de ascensores se encuentra al otro extremo, como se apreció en la figura 2. Este periodo podría corresponder a un modo torsional, con amplitudes máximas cerca de este eje, pero para poder asegurar esta hipótesis se deben realizar más estudios. No hay participación importante de frecuencias altas, algo que se esperaba debido a la desocupación del inmueble.

Con respecto a la calibración del modelo matemático por medio de mediciones de vibraciones ambientales (MVA), se tuvieron que tomar en cuenta varias consideraciones. Las cargas gravitacionales aplicadas al modelo (especialmente las cargas temporales) debieron ser las reales existentes, no cargas máximas de diseño. Además se consideraron todos los elementos estructurales como no agrietados debido a que el edificio es nuevo y no había sufrido algún sismo moderado o fuerte, por último y de mayor consecuencia, se tuvo que restringir la rotación (el grado de libertad asociado a la rotación) de las conexiones en todos los marcos y considerar las mismas como infinitamente rígidas. Siguiendo estas consideraciones se logró que el periodo fundamental $T 1$ estimado por el modelo fuera igual a $0.47 \mathrm{~s}$, conteniendo un error con respecto a las MVA igual al $4.4 \%$ para $\operatorname{los} \mathrm{EF}(T 1=0.45 \mathrm{~s})$ e igual a $6.8 \%$ para las FT $(T 1=0.44 \mathrm{~s})$.

\section{REFERENCIAS}

Almac, U, M Alaboz, I Bal, O Karahan y S Dashti (2016), "Structural observations on Macedonian tower, Edirne", En Van Balen \& Verstrynge (coords.), Structural Analysis of Historical Constructions. Londres, Taylor \& Francis Group. DOI: DOI: 10.1201/9781315616995-258

Andersen, P (sin fecha), Technical Review No. 1: Experimental Modal Analysis and Operational Modal Analysis. Obtenida el 1 de octubre de 2015, http://www.svibs.com/solutions/Technical_Reviews/Review_1_2012.aspx

Asmussen, J (1997), "Modal Analysis based on the Random Decrement Technique: Application to Civil Engineering Structures", Tesis de doctorado. Dinamarca, Departamento de Tecnología para Edificios e Ingeniería Estructural, Universidad de Aalborg.

Bendat, J S y A G Piersol (1993), Engineering Applications of Correlation and Spectral Analysis. Nueva York, John Wiley \& Sons.

Cantieni, R y T Biro (2005), "Office floor vibrations: modal parameter identification and vibration monitoring", Proceedings of the 6st European Conference on Structural Dynamics. Paris, Francia.

Carvajal, O (2005), "Identificación de parámetros dinámicos de dos tipos de edificio, utilizando técnicas analíticas y experimentales", Tesis de Grado. Universidad de Costa Rica, San José, Costa Rica.

Chang, C S (1975), Study of Dynamic Characteristics of Aeroelastic Systems Utilizing Randomdec Signatures. NASA-CR-132563.

Churilov, S, K Milkova y E Dumova (2016), "Dynamic identification analysis for the FE model updating of masonry buildings", En Modena, da Porto \& Valluzi (coords.), Brick and Block Masonry-Trends, Innovations and Challenges. Londres, Taylor \& Francis Group. DOI: 10.1201/b21889-17 
Demirtas, B, A Bayraktar y A Dumanoglu (2016), "Ambient Vibration Based Model Updating Effects on the Earthquake Response of Tall Buildings", 3rd International Balkans Conference on Challenges of Civil Engineering. 19-21 Mayo, Tirana, Albania.

Domínguez, A (2012), "Determinación de las propiedades dinámicas y efecto de la interacción sueloestructura de un edificio en la ciudad de México mediante un estudio de vibración ambiental”, Tesis de maestría. México, Escuela Superior de Ingeniería y Arquitectura U.Z., Instituto Politécnico Nacional.

Elyamani, A, P Roca, O Caselles y J Clapes (2017), "Seismic safety assessment of historical using updated numerical model: The case of Mallorca cathedral in Spain", Engineering Failure Analysis, Vol. 74, pp. 54-79. DOI: 10.1016/j.engfailanal.2016.12.017

Foti, D, M Diaferio, N Giannoccaro y M Mongelli (2016), "Ambient vibration testing, dynamic identification and model updating of a historic tower", NDT \& E International, Vol. 47, pp. 88-95. DOI: 10.1016/j.ndteint.2011.11.009

Günaydin, M, S Adanur, A Altunisik, B Sevim y A Bayraktar (2016), "Finite Modeling Updating Effects on the Dynamic Response of Building Models," Journal of Testing and Evaluation, Vol. 45, No. 5. Advance online publication. DOI: 10.1520/JTE20150515

Málaga, C y O Gómez (2007), "Caracterización Estructural por medio de Vibraciones Ambientales", XVI Congreso Nacional de Ingeniería Civil, Arequipa, Perú.

Michel, C, P Guéguen y P Bard (2008), "Dynamic parameters of structures extracted from ambient vibration measurements: an aid for the seismic vulnerability assessment of existing buildings in moderate seismic hazard regions", Soil Dynamics and Earthquake Engineering, Vol. 28, No. 8, pp. 593-604. DOI: 10.1016/j.soildyn.2007.10.002

Oliveira, C y M Navarro (2010), "Fundamental periods of vibration of RC buildings in Portugal from insitu experimental and numerical techniques", Bulletin of Earthquake Engineering, Vol. 8, No. 3, pp 609-642. DOI: 10.1007/s10518-009-9162-1

Murià, D, L Fuentes y R González (2000), "Uncertanties in the estimation of natural frequencies of buildings in Mexico city", 12 World Conference on Earthquake Engineering. Auckland, Nueva Zelanda.

Rodrigues, J y R Brincker (2005), “Application of the Random Decrement Technique in Operational Modal Analysis", Proceedings of the 1st International Operational Modal Analysis Conference. Copenhagen, pp. 191-200.

Schuster, N, C Ventura, A Felber y J Pao (1994), "Dynamic Characteristics of a 32 Story High-Rise Building during Construction", Proceedings Fifth U.S. National Conference on Earthquake Engineering, Vol. n, pp. 701-710.

Skrinar, M y A Strukelj (1996), "Eigenfrequency Monitoring during Bridge Erection", Structural Engineering International, Vol. 6, No. 33, pp. 191-194. DOI: 10.2749/101686696780495446

Tamura, Y, L Zhang, A Yoshida, S Nakata y T Itoh (2002), "Ambient Vibrations Tests and Modal Identification of Structures by FDD and 2DOF-RD Technique", Structural Engineers World Congress, Yokohama, Japón.

Ventura, C y N Schuster (1996), "Structural Dynamic Properties of a Reinforced Concrete High-Rise Building during Construction”, Canadian Journal of Civil Engineering, Vol. 23, No. 4, pp. 950-972. DOI: $10.1139 / 196-901$ 
Wathelet, M (2011), "Geopsy: Geophysical Signal Database for Noise Array Processing”, Programa computacional. Versión 2.9.0. Disponible en http://www.geopsy.org 